\title{
Comportamento da sutura palatina mediana em crianças submetidas à expansão rápida da maxila: avaliação mediante imagem de tomografia compu- tadorizada
}

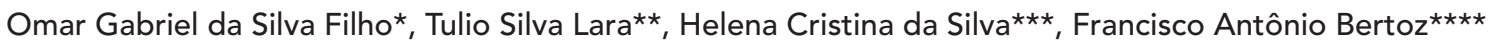

\section{Resumo}

Objetivo: o presente estudo, de caráter prospectivo, objetivou avaliar a imagem da sutura palatina mediana em crianças submetidas à expansão rápida da maxila por meio de tomografia computadorizada, após a fase de contenção. Metodologia: a amostra constou de 17 crianças de ambos os gêneros, na faixa etária compreendida entre 5 anos e 2 meses e 10 anos e 5 meses no início do tratamento, provenientes da Clínica de Ortodontia Interceptiva da Sociedade de Promoção Social do Fissurado Lábio-Palatal da Universidade de São Paulo (PROFIS-USP), Bauru/SP. Tomografias computadorizadas foram implementadas para avaliar o comportamento da sutura palatina mediana em diferentes momentos do tratamento. Resultados e Conclusões: constatou-se que, após um período médio de 8 a 9 meses de contenção com o aparelho expansor, a sutura palatina mediana mostrou-se completamente ossificada, desde a região da espinha nasal anterior até a espinha nasal posterior. Tal informação esclarece o comportamento da sutura palatina mediana frente à expansão rápida da maxila e reitera o senso comum quanto ao caráter biológico do procedimento.

Palavras-chave: Expansão maxilar. Tomografia computadorizada por raio-X. Ortodontia.

\section{INTRODUÇÃO}

A partir da década de 1960, Haas ${ }^{16,17,18,19}$ rubricou uma série de artigos referentes à mecânica transversal ortopédica aplicada no arco dentário superior, revelando os seus efeitos histológicos, clínicos e radiográficos induzidos pelo aparelho expansor fixo de ancoragem máxima, dento-muco-suportado. Embora distantes no tempo, estes trabalhos continuam sendo os mais influentes sobre o tratamento da deficiência maxilar. Há muito tempo, temos aplicado a filosofia da expansão ortopédica segundo Haas ${ }^{18}$, com considerável fidelidade, já que o aparelho usado na dentadura permanente é quase um clone do aparelho original publicado pelo autor ${ }^{4}$. Mas fomos além da proposta original, aplicando o aparelho ortopédi-

* Ortodontista do Hospital de Reabilitação de Anomalias Craniofaciais da Universidade de São Paulo, HRAC-USP, Bauru/SP.

* Aluno do Curso de Mestrado em Odontologia, Área de concentração Ortodontia da Faculdade de Odontologia de Araçatuba da Universidade Estadual Paulista, UNESP, Araçatuba/SP. Professor do Curso de Aperfeiçoamento em Ortodontia Preventiva e Interceptiva da Sociedade de Promoção Social do Fissurado Lábio-Palatal da Universidade de São Paulo, PROFIS-USP, Bauru/SP.

*** Professora Doutora do Hospital das Clínicas da Faculdade de Medicina de Marília, Marília/SP.

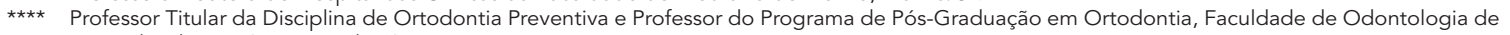
Araçatuba da UNESP, Araçatuba/SP. 
co transversal para os estágios de dentadura decídua e mista (Fig. 1) 32 . O critério indicativo para a expansão ortopédica passou a ser o diagnóstico estrutural da atresia, independentemente do estágio do desenvolvimento oclusal e que pode estar presente desde a dentadura decídua ${ }^{33}$.

O fato é que a expansão ortopédica nos estágios de dentadura decídua e mista pode parecer uma transgressão biológica, para quem não está habituado a esta terapia. Entretanto, para os muitos adeptos dessa filosofia, a prática tem comprovado que a expansão ortopédica em estágios que antecedem a dentadura permanente não induz iatrogenias irreversíveis ${ }^{30}$. O seu efeito permanente reduz-se a vantagens anatômicas e oclusais, como se comprova na figura 1 .

Quando acionado o parafuso, ocorre o afastamento das metades do aparelho, criando-se uma força equivalente à resistência da estrutura esquelética da face ${ }^{44}$, o que leva ao afastamento das metades maxilares ${ }^{9,14}$, abrindo a sutura palatina mediana de forma pendular no sentido horizontal. A sutura apresenta maior abertura na região da espinha nasal anterior (ENA) quando comparada à região da espinha nasal posterior (ENP) 10,18,34,41,43, em decorrência dos pilares posteriores de sustentação da maxila na base do crânio, como por exemplo os processos pterigóides do osso esfenóide.

O distanciamento dos processos maxilares provoca ainda o abaixamento da maxila, com possível deslocamento no sentido anterior ${ }^{15,20,31,36,43}$, induzindo no plano sagital a rotação horária da mandíbula ${ }^{5,6,18,20,29,31,36,42,43}$. Este comportamento maxilar no sentido sagital, entretanto, é reversível, não influenciando o padrão de crescimento facial $^{7,15,20,43}$.
Em artigo recente ${ }^{34}$, avaliamos a resposta da sutura palatina mediana durante a expansão rápida da maxila, com o aparelho expansor fixo tipo Haas, em crianças no estágio de dentadura decídua e mista, com tomografia computadorizada. As imagens tomográficas, no nível da sutura palatina mediana (Fig. 2), permitiram diagnosticar que, assim como a espinha nasal anterior, a espinha nasal posterior sofre o impacto da expansão rápida da maxila. As imagens mostraram claramente que a sutura palatina mediana também abriu na sua extremidade mais posterior, contígua aos vigorosos processos pterigóides do osso esfenóide - considerados pilares de resistência à disjunção maxilar. Essa particularidade ainda não havia sido exposta na literatura com material humano. Na maior parte dos pacientes, a sutura palatina mediana abriu de forma piramidal, mas em alguns essa abertura pareceu paralela.

A quantificação do efeito ortopédico sobre a sutura palatina mediana foi realizada com medições nas regiões mais anterior (espinha nasal anterior) e posterior (espinha nasal posterior). As medidas foram realizadas com ferramenta existente no programa de leitura da imagem tomográfica. A média e o desvio-padrão para essas distâncias, antes e após o procedimento de expansão rápida da maxila, podem ser vistos na tabela 1 . Pelos dados numéricos, a abertura da sutura palatina mediana no seu limite posterior equivale a $43 \%$ da abertura na extremidade anterior.

O resultado exposto na tabela 1 vem corroborar estudos esparsos realizados, que deixam entrever o envolvimento da extremidade posterior da maxila no processo de expansão rápida da mesma. Um dos estudos é biomecânico e usa como meto-

Tabela 1 - Média ( $\bar{x}$ ) e desvio-padrão (d.p.), em milímetros, para a distância aberta na região da espinha nasal anterior (ENA) e na região da espinha nasal posterior (ENP), nos estágios pré-tratamento e imediatamente após a expansão rápida da maxila (Fonte: SILVA FILHO et al. ${ }^{34}$ ).

\begin{tabular}{|c|c|c|c|c|c|}
\hline \multirow{2}{*}{ variáveis } & \multicolumn{2}{|c|}{ pré-expansão } & \multicolumn{2}{|c|}{ pós-expansão } & \multirow{2}{*}{$\begin{array}{c}\text { diferença média pré-expansão e } \\
\text { pós-expansão }\end{array}$} \\
\hline & $\overline{\mathbf{x}}$ & d.p. & $\overline{\mathbf{x}}$ & d.p. & \\
\hline ENA & 0,15 & 0,01 & 2,36 & 0,05 & 2,21 \\
\hline ENP & 0,15 & 0,01 & 1,10 & 0,04 & 0,95 \\
\hline
\end{tabular}



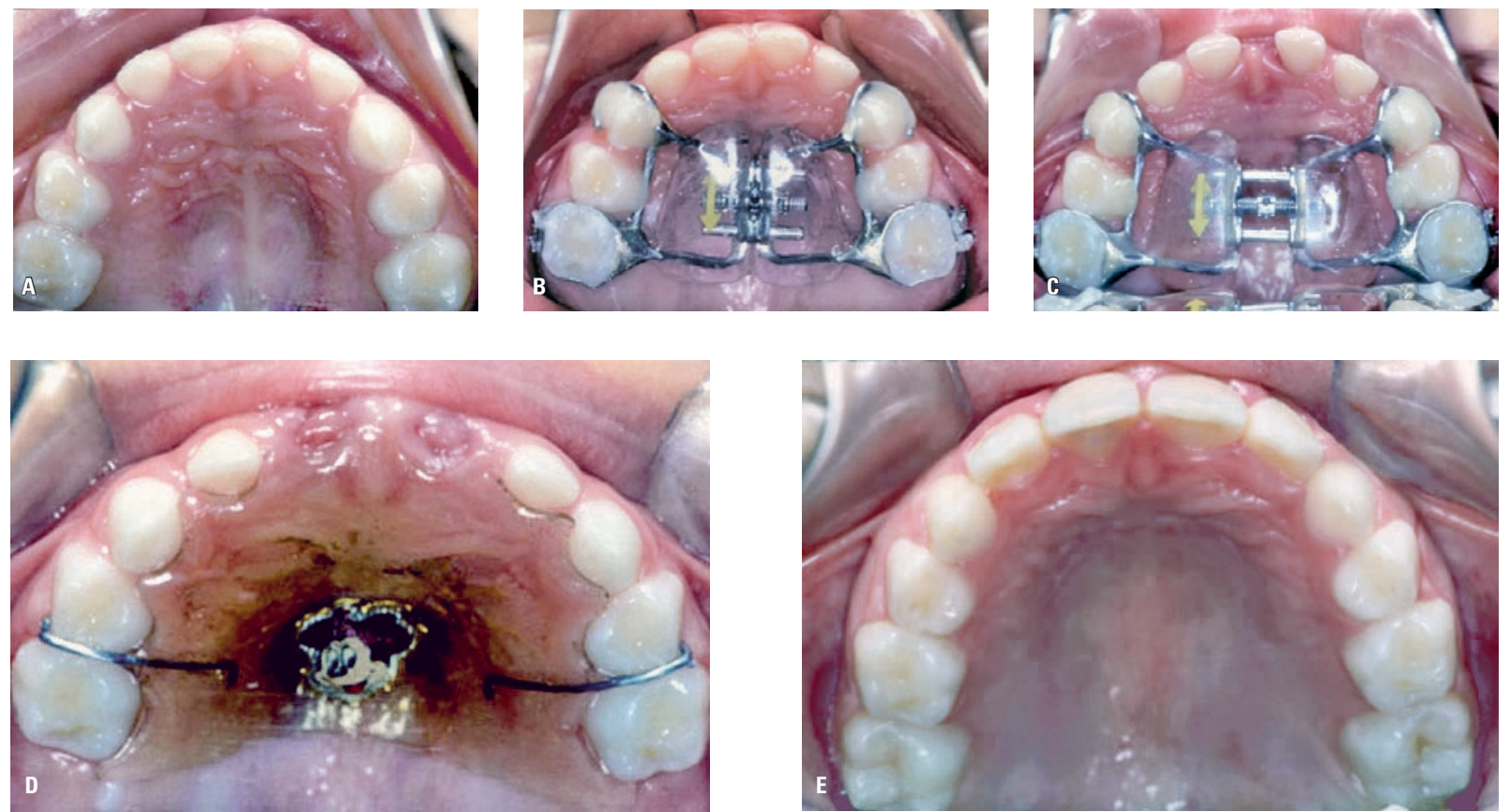

FIGURA 1 - Exemplo da expansão ortopédica aplicada no estágio de dentadura decídua. 0 aparelho expansor fixo tipo Haas modificado para as dentaduras decídua e mista, usando um protocolo de ativação rápida, uma volta completa por dia, distribuída igualmente pela manhã e pela noite, modificou a morfologia do arco dentário superior. Paciente A.A.: A) pré-tratamento; B) Haas instalado; C) pós-expansão; D) 5 meses pós-expansão (fase de contenção); E) morfologia do arco no período intertransitório da dentadura mista.

dologia uma duplicação tridimensional do esqueleto craniofacial com 12 anos de idade, onde se mostrou que, do ponto de vista oclusal, as duas metades maxilares separaram-se quase paralelamente 22 .

Esgotada a fase de ativação do parafuso expansor e finalizada a chamada fase ativa da expansão, inicia-se um período em que o aparelho é mantido passivamente na boca até a completa ossificação da sutura palatina mediana. Este processo de neoformação óssea, avaliado por meio da radiografia oclusal de maxila, pode variar por volta de três a seis meses, quando então, o aparelho expansor é removido e uma placa de contenção removível é instalada, sendo o paciente controlado por mais um ano (Fig. 1). Entretanto, o controle da ossificação da sutura palatina mediana por meio de radiografias oclusais de maxila não alcança a região mais posterior. A sobreposição de estruturas e o caráter bidimensional das imagens comprometem a avaliação da ossificação da sutura palatina me- diana em todo o seu trajeto ${ }^{13}$.

Neste sentido, a tomografia computadorizada se reveste de vantagens sobre as radiografias, uma vez que permite a visualização nítida de uma secção do corpo humano, sem a sobreposição de estruturas. Já ficou demonstrado, por meio do exame tomográfico, o que acontece na região da espinha nasal anterior e espinha nasal posterior imediatamente após a fase ativa da expansão. Resta-nos saber se a ossificação da sutura palatina mediana ocorre em toda sua extensão durante a fase de contenção com o aparelho expansor instalado.

\section{PROPOSIÇÃO}

Sabendo-se que o rompimento da sutura palatina mediana ocorre desde a espinha nasal anterior até a espinha nasal posterior ${ }^{34}$, o objetivo deste estudo consistiu em desvendar, por meio da tomografia computadorizada, se o processo de ossificação da sutura também ocorre em toda sua extensão. 

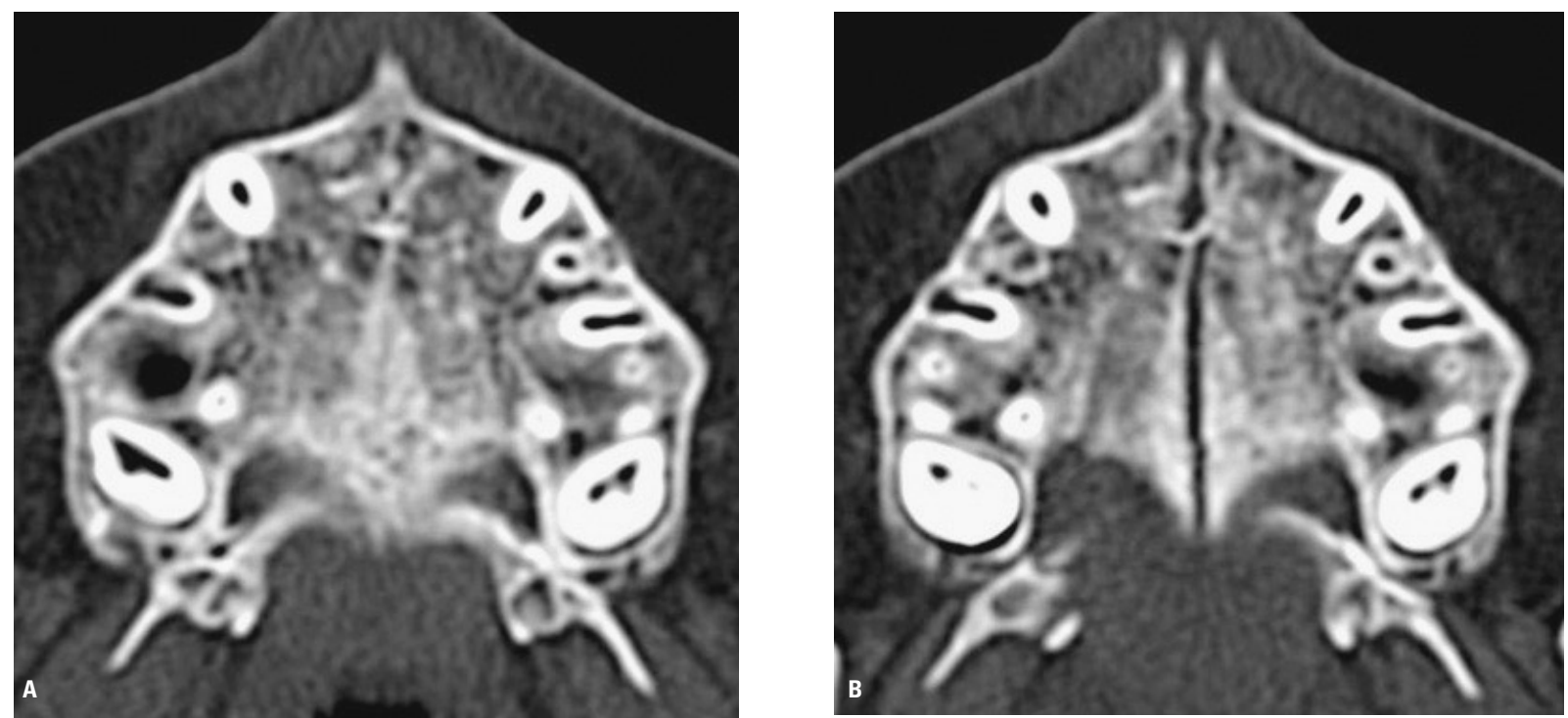

FIGURA 2 - Tomografias computadorizadas do paciente V.M.G. antes e imediatamente depois do procedimento de expansão ortopédica da maxila. A imagem mostra a ruptura da sutura palatina mediana em toda a sua extensão, desde a espinha nasal anterior até a espinha nasal posterior: A) pré-expansão e B) pós-expansão.

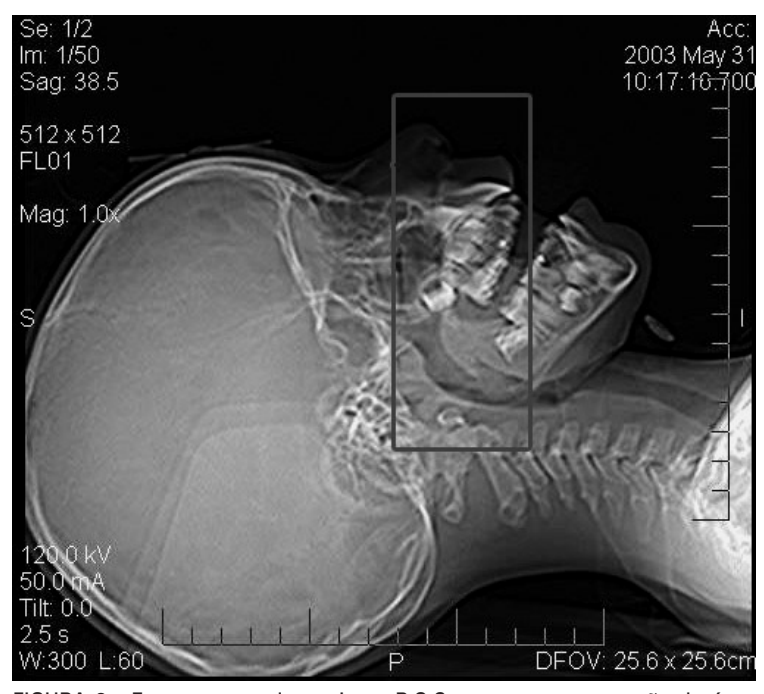

FIGURA 3 - Escanograma do paciente R.O.S. com a representação da área maxilar englobada no exame de tomografia computadorizada.

\section{MATERIAL}

A amostra utilizada no presente estudo constou de 17 das 18 crianças que compunham o primeiro trabalho ${ }^{34}$ em que avaliamos a expansão rápida da maxila por meio de tomografia computadorizada. As crianças, sendo 10 do gênero masculino e 7 do gênero feminino, apresentavam uma faixa etária de 5 anos e 2 meses a 10 anos e 5 meses, com idade média de 8 anos e 2 meses no início do tratamento. Estas foram triadas para tratamento ortodôntico na Clínica de Ortodontia Interceptiva da Sociedade de Promoção Social do Fissurado Lábio-Palatal da Universidade de São Paulo, PROFIS-USP, Bauru/ SP. Apenas um paciente encontrava-se no estágio de dentadura decídua, estando todos os demais no estágio de dentadura mista. Para o tratamento da atresia do arco dentário superior utilizou-se um aparelho expansor fixo dento-muco-suportado tipo Haas (Fig. 1). O protocolo de ativação ${ }^{6}$ consistiu em acionar o parafuso expansor uma volta completa por dia até a obtenção de uma boa morfologia do arco dentário superior, com alguma sobrecorreção.

\section{MÉTODOS \\ Tomografia computadorizada}

Para estudar o comportamento da sutura palatina mediana frente à expansão rápida da maxila, utilizou-se a tomografia computadorizada (Fig. 2, 3,4 ), um método de diagnóstico por imagem que permite obter a visualização nítida de uma secção do corpo humano nos três planos do espaço. Todos os pacientes foram submetidos ao exame tomográfico computadorizado três vezes: antes da 
expansão, imediatamente após a fase ativa da expansão e após a fase de contenção com o aparelho expansor. Foi empregado o aparelho de tomografia computadorizada helicoidal Toshiba, modelo Xvision EX (Toshiba Corporation Medical Systems Company, Otawara-Shi, Japão), fabricado em 1997, trabalhando com 120KV e 100mA, com colimador quadrangular de $0,9 \mathrm{~mm} \times 0,9 \mathrm{~mm}$, inexistindo a radiação secundária.

\section{Corte axial paralelo ao plano palatino na altu- ra da sutura palatina mediana}

Foram realizados cortes axiais de $1 \mathrm{~mm}$ de espessura, paralelamente ao plano palatino, englobando as regiões dentoalveolar e basal da maxila, até o terço inferior da cavidade nasal (Fig. 4). A extensão escaneada totalizou, aproximadamente, de $36 \mathrm{~mm}$ a $40 \mathrm{~mm}$, portanto, de 36 a 40 cortes. Os dados foram enviados a uma workstation (Silicon Graphics, Toshiba Corporation Medical Systems Company, Otawara-Shi, Japão), ou estação de trabalho independente. Nesse computador, desvinculado do aparelho de tomografia computadorizada que adquiriu as imagens, o software Alatoview (Toshiba Corporation Medical Systems Company) possibilitou a execução de reconstruções de cortes axiais paralelos ao plano palatino ao nível da sutura palatina mediana (Fig. 4).

Os cortes tomográficos foram avaliados morfologicamente pelos autores que, em comum acordo, diagnosticaram o comportamento da sutura palatina mediana após a fase de contenção, por volta de 8 a 9 meses com o aparelho expansor fixo. Além do diagnóstico visual, a distância aberta no extremo anterior da sutura palatina me- diana e a região da espinha nasal posterior foram medidas com auxílio de ferramenta de medição existente no próprio programa para a leitura das tomografias. Foram calculados a média e o desviopadrão para a abertura da sutura palatina mediana no seu extremo anterior e na região da ENP, bem como a diferença para cada uma delas no período pré-expansão e pós-contenção. Foi aplicado o teste $t$ de Student, a fim de averiguar se havia ou não diferença estatisticamente significante para os valores medidos na região da ENA e ENP na fase de pré-expansão e pós-contenção (Tab. 2).

\section{RESULTADOS E DISCUSSÃO}

A avaliação qualitativa dos cortes tomográficos pós-contenção demonstrou normalidade ao longo da sutura palatina mediana, como ilustra a figura 4. A nova sutura se organizou e as imagens tomográficas pós-contenção das 17 crianças mostraram semelhança com as imagens pré-tratamento. $\mathrm{O}$ comportamento da sutura palatina mediana durante as fases ativa e passiva da expansão rápida da maxila pode ser deduzido a partir da figura 4. Quanto à avaliação quantitativa, a tomografia computadorizada pós-contenção mostrou que apenas na região da espinha nasal anterior foi encontrada uma pequena distância entre as margens suturais $(0,28 \mathrm{~mm})$. Entretanto, esta abertura não é estatisticamente significante quando comparada à distância apresentada nesta região na fase pré-expansão $(0,15 \mathrm{~mm})$ (Tab. 2). Os resultados desvendaram, com maior nitidez em relação à imagem obtida em radiografia oclusal de maxila, o comportamento da sutura palatina mediana frente à expansão rápida da maxila. A sutura mostrou-se

Tabela 2 - Média ( $\bar{x}$ ) e desvio-padrão (d.p.), em milímetros, para a distância entre as lâminas palatinas na região da ENA e ENP, nos estágios pré-tratamento, pós-expansão e pós-contenção com 0 aparelho expansor. Ainda, a diferença média e 0 valor $p$ para as distâncias entre as lâminas palatinas na região da ENA e ENP nas fases pré-expansão e pós-contenção.

\begin{tabular}{|c|c|c|c|c|c|c|c|c|}
\hline \multirow{2}{*}{ variáveis } & \multicolumn{2}{|c|}{ pré-expansão } & \multicolumn{2}{|c|}{ pós-expansão } & \multicolumn{2}{|c|}{ pós-contenção } & \multirow{2}{*}{$\begin{array}{c}\text { diferença média pré-expansão } \\
\text { e pós-contenção }\end{array}$} & \multirow{2}{*}{$\mathbf{p}$} \\
\hline & $\overline{\mathbf{x}}$ & d.p. & $\overline{\mathbf{x}}$ & d.p. & $\overline{\mathbf{x}}$ & d.p. & & \\
\hline ENA & 0,15 & 0,01 & 2,36 & 0,05 & 0,28 & 0,02 & $+0,13$ & 0,2318 n.s. \\
\hline ENP & 0,15 & 0,01 & 1,10 & 0,04 & 0 & 0 & $-0,15$ & 0,0315 n.s. \\
\hline
\end{tabular}

n.s. = estatisticamente não significante, ao nível de significância de $5 \%$. 

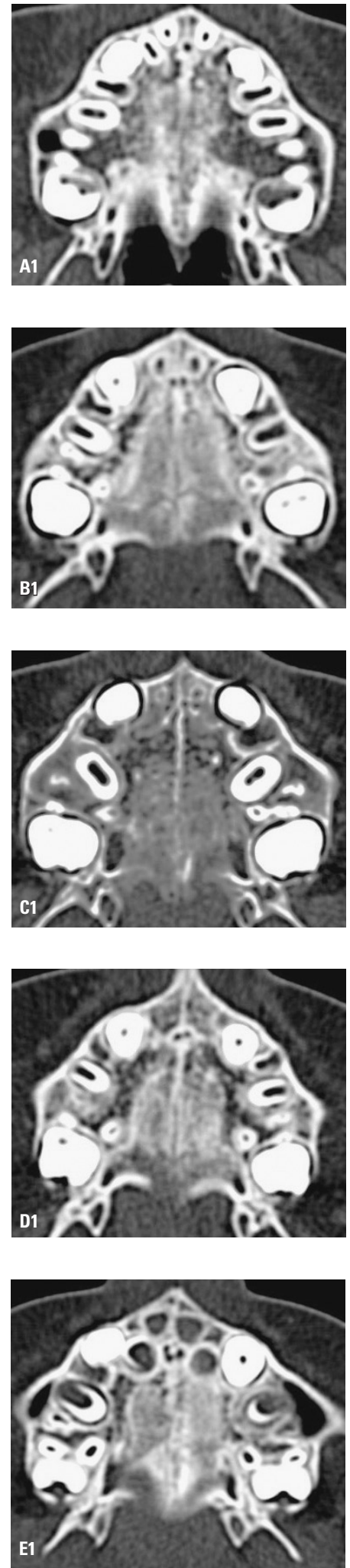
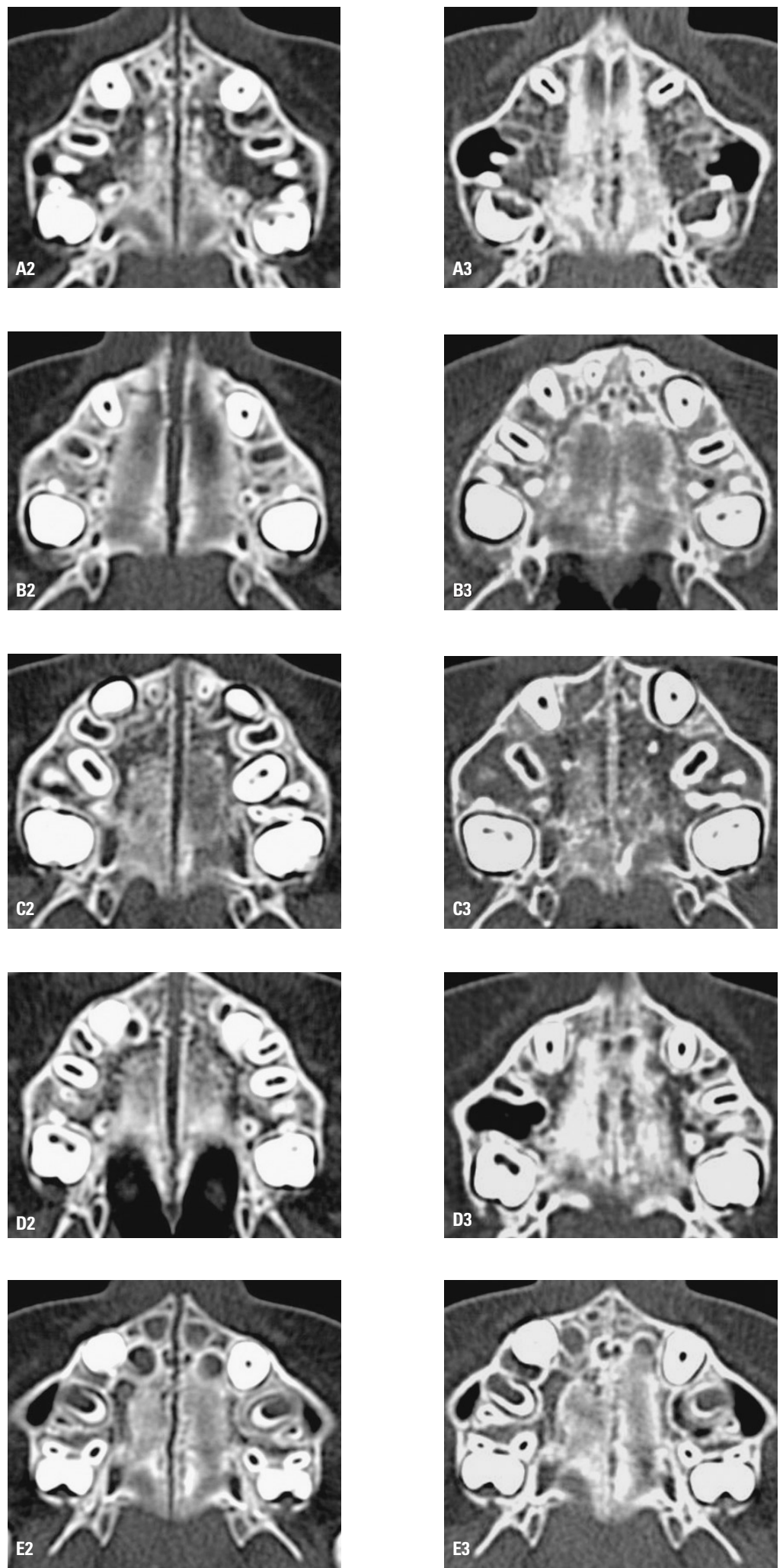

FIGURA 4 - Imagens das suturas palatinas medianas diagnosticadas nos exames tomográficos (corte axial ao nível da abóbada palatina) pré-expansão (Coluna 1) pós-expansão (Coluna 2) e pós-contenção com o aparelho expansor (Coluna 3) de 5 pacientes selecionados aleatoriamente da amostra total. 
inteiramente ossificada após o período aproximado de 8 a 9 meses de contenção.

A sutura palatina mediana é rompida durante a fase ativa do processo de expansão ortopédica da maxila, à medida que o parafuso expansor é acionado; mas se reorganiza em poucos meses, durante a fase passiva do processo de expansão ortopédica, mediante reparo do tecido conjuntivo e formação de novo osso ${ }^{23}$. Na clínica ortodôntica, a ruptura da sutura palatina mediana, bem como a sua calcificação subseqüente, são acompanhadas pela radiografia oclusal de maxila, que identifica claramente o distanciamento dos processos maxilares e o subseqüente processo de ossificação paulatina na região mais anterior do palato. As margens da sutura velha, distanciadas com a disjunção maxilar, vão desaparecendo, simultaneamente, com o surgimento das margens da sutura neoformada. É coerente admitir que, do ponto de vista de estabilidade em longo prazo, o aparelho expansor fixo só deve ser removido depois de comprovada a total neoformação óssea da sutura palatina mediana, visto que há uma tendência de recidiva da nova morfologia do arco dentário superior $24,25,35,39,40$. Em um estudo retrospectivo ${ }^{35}$, reunindo 17 pacientes tratados com expansão rápida da maxila na dentadura permanente e acompanhados até 5 anos pós-tratamento, os autores observaram uma diminuição nas distâncias transversais medidas em modelos de gesso (Tab. 3). No entanto, os autores ressaltaram que a recidiva documentada numericamente não trouxe conseqüências apreciáveis na relação intra-arco e interarcos para as oclusões tratadas. Embora vários fatores estejam envolvidos no fenômeno da recidiva, foi aventada a hipótese da restituição sutural ajudar na estabilidade pós-expansão ${ }^{24}$.

Hoje, não há dúvida sobre o que acontece com a sutura palatina mediana no processo de expansão rápida da maxila. A separação dos processos maxilares foi inferida, inicialmente, pelo precursor do processo, o dentista americano Angell ${ }^{1,2}$, com base no diastema que se abriu entre os incisivos centrais superiores, já que não havia sido desco- berto ainda o raio-x. Quase 100 anos mais tarde, Haas $^{19}$ comentou que o procedimento, tal qual é aceito atualmente, com ancoragem pesada, foi reintroduzido na Ortodontia dos Estados Unidos sob a influência da visita do professor Korkhaus na Universidade de Illinois, na década de 1950, quando a expansão ortopédica passou a ser objeto de estudo. Surgiu o primeiro estudo animal feito com porcos Duroc-Poland-China, para comprovar a possibilidade de abertura da sutura palatina mediana em até $15 \mathrm{~mm}$, usando ancoragem pesada para promover o efeito ortopédico sugerido por Angell ${ }^{1}$. Os resultados dessa tese foram notificados em artigo publicado por $\mathrm{Haas}^{18}$, no ano de 1961, e comprovaram a abertura da sutura palatina mediana, seguida de neoformação óssea.

De fato, trabalhos prévios às publicações de Haas $^{18}$ haviam cogitado a possibilidade de abertura da sutura palatina mediana em gatos ${ }^{10} \mathrm{e}$ em cães ${ }^{11}$. No entanto, os aparelhos usados empregavam ancoragem insuficiente para promover o tão almejado efeito ortopédico. Mesmo assim, esses estudos revelaram abertura da sutura palatina mediana de $0,7 \mathrm{~mm}$ na região anterior, sem efeito na região posterior do palato. Comentaram, ainda, que a abertura discreta da sutura palatina mediana foi acompanhada pela formação de novo osso nas margens suturais.

Um novo estudo histológico ${ }^{9}$ repetiu o trabatho de $\mathrm{Haas}^{18}$ em macacos, comprovando a ruptura da sutura palatina mediana e mostrando a neoformação óssea progressiva subseqüente. No momento da ruptura, observou-se a área do defeito ósseo preenchida com tecido conjuntivo fibroso desorganizado, bem vascularizado, sugestivo de resposta inflamatória crônica suave. Depois de 3 meses, a sutura apresentou um aspecto histológico similar aos macacos controle, mas o osso adjacente era irregular e muito celularizado, não obedecendo o padrão lamelar usual do osso mais distante da área sutural, indicando haver uma formação óssea rápida. $\mathrm{O}$ aspecto histológico sugeria remodelação da sutura recém-reparada. 
Tabela 3 - Valores médios e desvios-padrão para as medidas transversais obtidas dos modelos de gesso do arco dentário superior (Fonte: SILVA FILHO et al. ${ }^{35}$ ).

\begin{tabular}{|c|c|c|c|c|c|c|}
\hline \multirow{2}{*}{ dentes } & \multicolumn{2}{|c|}{ pós-tratamento } & \multicolumn{2}{|c|}{ pós-contenção (5 anos) } & \multirow{2}{*}{$\begin{array}{c}\begin{array}{c}\text { diferença } \\
\text { contenção e } \\
\text { tratamento }\end{array} \\
\overline{\mathbf{x}}\end{array}$} & \multirow{2}{*}{ teste $t$} \\
\hline & $\overline{\mathbf{x}}$ & d.p. & $\overline{\mathbf{x}}$ & d.p. & & \\
\hline $3-3$ & $35,51 \mathrm{~mm}$ & $1,65 \mathrm{~mm}$ & $34,86 \mathrm{~mm}$ & $1,74 \mathrm{~mm}$ & $-0,64 \mathrm{~mm}$ & $0,0019^{*}$ \\
\hline $4-4$ & $43,37 \mathrm{~mm}$ & $1,91 \mathrm{~mm}$ & $42,46 \mathrm{~mm}$ & $2,20 \mathrm{~mm}$ & $-0,91 \mathrm{~mm}$ & $0,0295^{*}$ \\
\hline $5-5$ & $48,96 \mathrm{~mm}$ & $2,09 \mathrm{~mm}$ & $47,73 \mathrm{~mm}$ & $3,0 \mathrm{~mm}$ & $-1,32 \mathrm{~mm}$ & $0,0205^{*}$ \\
\hline $6-6$ & $53,07 \mathrm{~mm}$ & $2,94 \mathrm{~mm}$ & $52,53 \mathrm{~mm}$ & $2,93 \mathrm{~mm}$ & $-0,54 \mathrm{~mm}$ & 0,0684 n.s. \\
\hline $7-7$ & $58,74 \mathrm{~mm}$ & $3,60 \mathrm{~mm}$ & $59,04 \mathrm{~mm}$ & $3,73 \mathrm{~mm}$ & $0,30 \mathrm{~mm}$ & 0,9741 n.s. \\
\hline
\end{tabular}

n.s. = estatisticamente não significante, ${ }^{*}$ nível de significância de $5 \%$.

Portanto, aos 3 meses, a morfologia sutural, quando avaliada radiograficamente, permanecia desorganizada e a área pobremente mineralizada, quando comparada com o grupo controle. Após 6 meses da fase ativa da expansão, a sutura mostrou-se bem organizada e histologicamente normal, mas o osso adjacente era de natureza irregular e não seguia o padrão lamelar normal. Somente no animal sacrificado com 9 meses, a região sutural mostrou-se com grau de mineralização semelhante aos animais do grupo controle. Outros trabalhos comprovaram a ossificação da sutura palatina mediana durante a fase do procedimento de expansão ortopédica ${ }^{3,10,12,21,26,27,28,37,38}$.

Kanekawa e Shimizu ${ }^{23}$, em 1998, estudaram a capacidade regenerativa da sutura palatina mediana, depois da disjunção maxilar em ratos, com a intenção de determinar a influência da idade sobre este fenômeno. A capacidade formativa da matriz óssea, em resposta ao estímulo provocado pelo estiramento mecânico decorrente da expansão orto- pédica, independe da idade, enquanto a velocidade de formação óssea é dependente da idade, sofrendo redução após a adolescência. Inferem a necessidade de mais tempo de contenção em pacientes adultos submetidos à expansão rápida da maxila.

O único trabalho histológico em humanos com o objetivo de estudar o comportamento da sutura palatina mediana foi realizado em crianças de 8 a 13 anos de idade ${ }^{26}$. Na terceira e quarta semanas após a expansão, conjuntamente ao alargamento da sutura, o tecido mostrou indícios de inflamação com acentuada atividade osteoblástica ao longo da superfície e processos ósseos. Após 5 a 6 semanas, a ossificação progrediu, exibindo ilhas ósseas ao longo da sutura. Já um ano após a expansão, a sutura completamente calcificada mostrava a formação das pontes ósseas nas margens suturais.

Do ponto de vista clínico, o controle da organização sutural entre os maxilares previamente separados é feito mediante o emprego da radiografia oclusal total de maxila (Fig. 5). A reestruturação
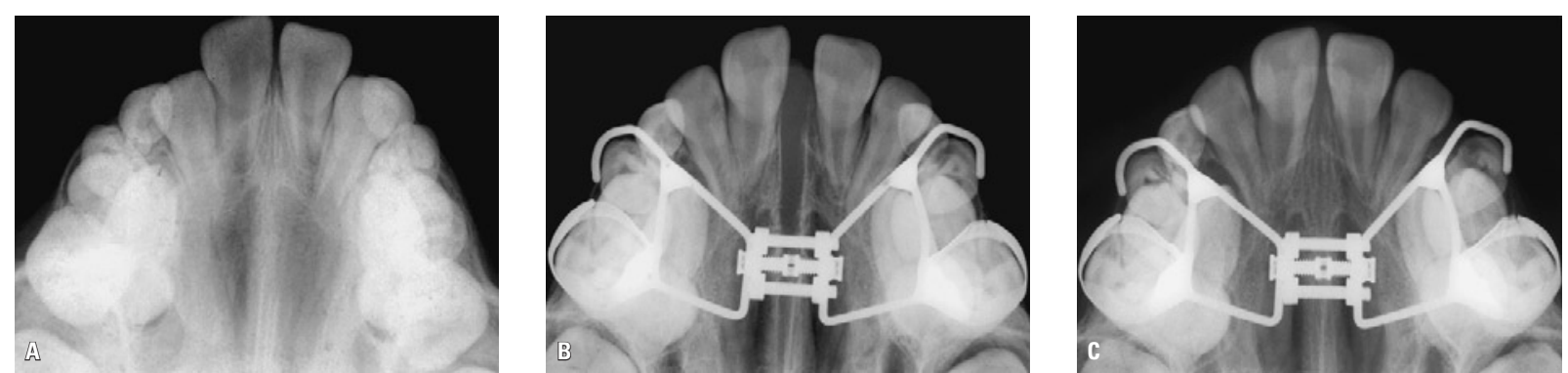

FIGURA 5 - Radiografias oclusais totais de maxila mostrando o comportamento da sutura palatina mediana num procedimento de expansão rápida da maxila: A) prétratamento (21/12/00); B) após a fase ativa (19/09/01) e C) após a fase passiva com o aparelho expansor (19/12/01). 
da sutura palatina mediana na imagem radiográfica oclusal é um indício de que o aparelho expansor pode ser removido. O processo de reparação óssea tem início logo depois da fase ativa da expansão rápida ${ }^{8,9,10,19}$, muito embora a ossificação plena da sutura palatina mediana, identificada na imagem da radiografia oclusal total de maxila, leve em torno de 5 meses. Este constitui o período de tempo no qual o aparelho permanece na boca, compreendendo a fase passiva da expansão rápida da maxila. O intervalo de três meses sugerido por Haas ${ }^{19}$ para a ossificação completa da sutura aberta não se comprova, na maioria dos casos, nas imagens com radiografias. Em decorrência dessa premissa, as crianças da presente pesquisa foram submetidas à última tomografia (imediatamente após a suspensão do aparelho expansor fixo) num período médio de 8 a 9 meses. As imagens tomográficas avaliadas imediatamente depois da suspensão do aparelho expansor revelam integridade sutural em toda extensão da sutura palatina mediana. $\mathrm{O}$ que não se consegue identificar por completo na imagem radiográfica pode ser comprovado na tomografia computadorizada, como se observa nas imagens tomográficas pré-expansão, pós-expansão e pós-contenção (Fig. 4). As imagens da figura 4 comprovam a ossificação da sutura palatina mediana em toda a sua extensão, desde a espinha nasal anterior até a espinha nasal posterior.

\section{CONCLUSÃO}

A imagem tomográfica englobando as regiões dentoalveolar e basal da maxila revelou que após 8 a 9 meses, em média, de contenção com o aparelho expansor, a sutura palatina mediana mostrou-se completamente ossificada em toda a sua extensão.

\title{
Behavior of the midpalatal suture in children submitted to rapid maxillary expan- sion: evaluation with computerized tomography
}

\begin{abstract}
Aim: The aim of this prospective study was to evaluate the midpalatal suture in children submitted to rapid palatal expansion with computerized tomography. Methods: The sample was comprised of 17 children aged between 5 years and 2 months and 10 years and 5 months, that were submited to rapid palatal expansion. The radiologic aspect of the sutures were evaluated through computerized tomography. Results and Conclusions: The tomographic images showed that, after the retention phase (8 to 9 months post-expansion), the midpalatal suture was completely ossified from the anterior to the posterior nasal spine region.
\end{abstract}

Key words: Palatal expansion technique. X-ray computed tomography. Orthodontics.

\section{REFERÊNCIAS}

1. ANGELL, E. H. Treatment of irregularity of the permanent or adult teeth. I. Dental Cosmos, Philadelphia, v. 1, no. 10, p. 540-544, May 1860

2. ANGELL, E. H. Treatment of irregularity of the permanent or adult teeth. II. Dental Cosmos, Philadelphia, v. 1, no. 10, p. 599-601, June 1860.

3. BRIN, I. et al. Rapid palatal expansion in cats: effect of age on sutural cyclic nucleotides. Am. J. Orthod., St. Louis, v. 79, no. 2, p. 162-175, Feb. 1981.
4. CAPELOZZA FILHO, L.; SILVA FILHO, O. G. Expansão rápida da maxila: considerações e aplicações clínicas. In: INTERLANDI, S. (Coord.). Ortodontia. 4. ed. São Paulo: Artes Médicas, 1999. p. 287-328.

5. CAPELOZZA FILHO, L.; SILVA FILHO, O. G. Expansão rápida da maxila: considerações gerais e aplicação clínica. Parte I. Rev. Dental Press Ortodon. Ortop. Maxilar, Maringá, v. 2, n. 3, p. 88-102, maio/jun. 1997.

6. CAPELOZZA FILHO, L.; SILVA FILHO, O. G. Expansão rápida da maxila: considerações gerais e aplicação clínica. Parte II. Rev. Dental Press Ortodon. Ortop. Maxilar, Maringá, v. 2, n. 4, p. 86-108, jul./ago. 1997. 
7. CHANG, J. Y.; McNAMARA JR., J. A.; HERBERGER, T. A. A longitudinal study of skeletal side effects induced by rapid maxillary expansion. Am. J. Orthod. Dentofacial Orthop., St. Louis, v. 112, no. 3, p. 330-337, Sept. 1997.

8. CLEALL, J. F. Growth of the palate and maxillary dental arch. J. Dent. Res., Chicago, v. 53, no. 5, p. 1226-1234, Sept./Oct. 1974

9. CLEALL, J. F. et al. Expansion of the midpalatal suture in the monkey. Angle Orthod., Appleton, v. 35, no. 1, p. 23-35, Jan. 1965.

10. DEBBANE, E. F. A cephalometric and histologic study of the effect of orthodontic expansion of the midpalatal suture of the cat. Am. J. Orthod., St. Louis, v. 44, no. 3, p. 187-218, Mar. 1958.

11. DEWEY, M. Development of the maxillae with reference to opening the median suture. Items Interest, Philadelphia, v. 35, p. $189-208,271-282,1913$

12. EKSTROM, C.; HENRIKSON, C. O.; JENSEN, R. Mineralization in the midpalatal suture after orthodontic expansion. Am. J. Orthod., St. Louis, v. 71, no. 4, p. 449-455, Apr. 1977.

13. ENNES, J.; CONSOLARO, A. Sutura palatina mediana: avaliação do grau de ossificação em crânios humanos. Rev. Dental Press Ortodon. Ortop. Facial, Maringá, v. 9, n. 5, p. 64-73, set./out. 2004.

14. GARDNER, G. E.; KRONMAN, J. H. Cranioskeletal displacements caused by rapid palatal expansion in the rhesus monkey. Am. J. Orthod., St. Louis, v. 59, no. 2, p. 146-155 Feb. 1971.

15. GARIB, D. G.; HENRIQUES, J. F. C.; JANSON, G. R. P. Avaliação cefalométrica longitudinal das alterações produzidas pela expansão rápida da maxila. Rev. Dental Press Ortodon. Ortop. Facial, Maringá, v. 6, n. 5, p. 17-30, set./ out. 2001.

16. HAAS, A. J. Long-term post treatment evaluation of rapid palatal expansion. Angle Orthod., Appleton, v. 50, no. 3, p. 189-217, July 1980.

17. HAAS, A. J. Palatal expansion: just the beginning of dentofacial orthopedics. Am. J. Orthod., St. Louis, v. 57, no. 3, p. 219-255, Mar. 1970

18. HAAS, A. J. Rapid expansion of the maxillary dental arch and nasal cavity by opening the midpalatal suture. Angle Orthod., Appleton, v. 31, no. 2, p. 73-90, 1961.

19. HAAS, A. J. The treatment of maxillary deficiency by opening the midpalatal suture. Angle Orthod., Appleton, v. 35, p. 200-217, July 1965.

20. HEFLIN, B. M. A three-dimensional cephalometric study of the influence of expansion of the midpalatal suture on the bones of the face. Am. J. Orthod., St. Louis, v. 57, no. 2, p. 194-195, Feb. 1970.

21. INOUE, N. et al. Radiographic observation of rapid expansion of human maxilla. Bull. Tokyo Med. Dent. Univ., Tokyo, v. 17, no. 3, p. 249-261, Sept. 1970.

22. ISERI, H. et al. Biomechanical effects of rapid maxillary expansion on the craniofacial skeleton, studied by the finite element method. Eur. J. Orthod., London, v. 20, no. 4, p. 347-356, Aug. 1998.

23. KANEKAWA, M.; SHIMIZU, N. Age-related changes on bone regeneration in midpalatal suture during maxillary expansion in the rat. Am. J. Orthod. Dentofacial Orthop., St. Louis, v. 114 , no. 6, p. 646-653, Dec. 1998

24. KREBS, A. Midpalatal suture expansion studies by the implant method over a seven-year period. Rep. Congr. Eur. Orthod. Soc., [s.I.], v. 40, p. 131-142, 1964.

25. LINDER-ARONSON, S.; LINDGREN, J. The skeletal and dental effects of rapid maxillary expansion. Br. J. Orthod., London, v. 6, no. 1, p. 25-29, Jan. 1979.

26. MELSEN, B. A histological study of the influence of sutural morphology and skeletal maturation on rapid palatal expansion in children. Trans. Eur. Orthod. Soc., [s.I.], p. 499-507, 1972.

27. MURRAY, J. M.; CLEALL, J. F. Early tissue response to rapid maxillary expansion in the midpalatal suture of the rhesus monkey. J. Dent. Res., Chicago, v. 50, no. 6, p. 1654-1660, Nov./Dec. 1971.
28. OHSHIMA, O. Effect of lateral expansion force on the maxillary structure in cynomolgus monkey. J. Osaka Dent. Univ., Osaka, v. 6, no. 1, p. 11-50, Apr. 1972.

29. SANDIKCIOGLU, M.; HAZAR, S. Skeletal and dental changes after maxillary expansion in the mixed dentition. Am. J. Orthod. Dentofacial Orthop., St. Louis, v. 111, no. 3, p. 321327, Mar. 1997.

30. SILVA FILHO, O. G.; FREITAS, P. Z.; SILVA, V. B. Aparelho expansor fixo dentomucossuportado: considerações clínicas sobre a mucosa palatina. Rev. Clin. Ortodon. Dental Press, Maringá, v. 3, n. 1, p. 57-63, fev./mar. 2004.

31. SILVA FILHO, O. G.; VILAS BOAS, M. C.; CAPELOZZA FILHO, $\mathrm{L}$. Rapid maxillary expansion in the primary and mixed dentitions: a cephalometric evaluation. Am. J. Orthod. Dentofacial Orthop., St. Louis, v. 100, no. 2, p. 171-179, Aug. 1991.

32. SILVA FILHO, O. G. et al. Correção da mordida cruzada posterior nas dentaduras decídua e mista. Rev. Assoc. Paul. Cir. Dent., São Paulo, v. 54, n. 2, p. 142-147, mar./abr. 2000

33. SILVA FILHO, O. G. et al. Epidemiologia da mordida cruzada posterior na dentadura decídua. J. Brás. Odontopediatr.

Odontol. Bebê, Curitiba, v. 6, n. 29, p. 61-68, jan./fev. 2003.

34. SILVA FILHO, O. G. et al. Evaluation of the midpalatal suture during rapid palatal expansion in children: a CT study. J. Clin. Pediatr. Dent., Birmingham, v. 29, no. 3, p. 231-238, Spring 2005.

35. SILVA FILHO, O. G. et al. Expansão rápida da maxila: comportamento transversal do arco dentário superior durante e 5 anos após o tratamento ortodôntico. Ortodontia, São Paulo, v. 37, n. 1, p. 58-69, jan./abr. 2004

36. SILVA FILHO, O. G. et al. Expansão rápida da maxila na dentadura permanente: avaliação cefalométrica. Ortodontia, São Paulo, v. 27, n. 2, p. 68-76, maio/ago. 1994.

37. SIMÕES, F. X. P. C.; ARAÚJO, T. M.; BITTENCOURT, M. A. V. Avaliação da maturação óssea na sutura palatina mediana, após expansão rápida da maxila, por meio da imagem digitalizada. Rev. Dental Press Ortodon. Ortop. Facial, Maringá, v. 8, n. 1, p. 59-67, jan./fev. 2003.

38. STARNBACH, $\mathrm{H}$. et al. Facioskeletal and dental changes resulting from rapid maxillary expansion. Angle Orthod., Appleton, v. 36, n. 2, p. 152-164, Apr. 1966.

39. TIMMS, D. J. Long-term follow-up of cases treated by rapid maxillary expansion. Trans. Eur. Orthod. Soc., [s.l.], v. 52, p. $211-215,1976$.

40. VARDIMON, A. D.; GRABER, T. M.; VOSS, L. R. Stability of magnetic versus mechanical palatal expansion. Eur. J. Orthod., London, v. 11, no. 2, p. 107-115, May 1989.

41. VARDIMON, A. D. et al. Magnetic versus mechanical expansion with different force thresholds and points of force application. Am. J. Orthod. Dentofacial Orthop., St. Louis, v. 92, no. 6 , p. 455-466, Dec. 1987

42. WERTZ, R.; DRESKIN, M. Midpalatal suture opening: a normative study. Am. J. Orthod., St. Louis, v. 71, no. 4, p. 367-381, Apr. 1977.

43. WERTZ, R. A. Skeletal and dental changes accompanying rapid midpalatal suture opening. Am. J. Orthod., St. Louis, v. 58, no. 1, p. 41-66, July 1970.

44. ZIMRING, J. F.; ISAACSON, R. J. Forces produced by rapid maxillary expansion. 3. Forces present during retention. Angle Orthod., Appleton, v. 35, p. 178-186, July 1965.

\section{Endereço para correspondência}

Omar Gabriel da Silva Filho

Rua: Sílvio Marchione, 3-20, Vila Universitária

CEP:17.043-900, Bauru / SP

E-mail: ortoface@travelnet.com.br 